\title{
Recent Diagnostic Techniques in Mycology
}

Malhotra $\mathbf{S}^{1 *}$, Sharma $\mathbf{S}^{1}$, Bhatia NJK ${ }^{1}$, Kumar $\mathbf{P}^{1}$, Bhatia NK ${ }^{2}$, Patil $\mathbf{V}^{3}$ and Hans $\mathbf{C}^{1}$

${ }^{1}$ Department of Microbiology, PGIMER and Associated RML Hospital, CCRUM, India

${ }^{2}$ Department of Orthodontics, Santosh Medical College, India

3 Jeevan Sakshi Hospital, India

*Corresponding author: Malhotra S, Department of Microbiology, PGIMER and associated RML Hospital, CCRUM, India, Tel: 7428177617; E-mail: drshalinimalhotra@yahoo.com

Rec Date: April 10, 2014, Acc date: July 14, 2014, Pub date: July 16, 2014

Copyright: ( 2014 Malhotra S, et al. This is an open-access article distributed under the terms of the Creative Commons Attribution License, which permits unrestricted use, distribution, and reproduction in any medium, provided the original author and source are credited.

\begin{abstract}
The frequency of fungal infections, especially of Invasive Fungal Infections (IFIs), has risen dramatically in recent years, and has high morbidity and mortality. Early and accurate diagnosis is important for timely implementation of antifungal therapy and decreasing the unnecessary use of toxic antifungal agents. Conventional microbiological, histological and radiological techniques remain the cornerstone of diagnosis but are insensitive and have a limited impact on clinical decision-making. There is always a need to characterize fungi quickly and accurately which can be done by rapid diagnostic techniques like antigen detection and molecular techniques. This is a brief review of all the microbiological techniques- conventional and recent alternative techniques especially elaborating antigen detection and molecular techniques which are available for diagnosis of fungal infections.
\end{abstract}

Keywords: Invasive fungal infections; Rapid diagnostic techniques; Molecular techniques

\section{Introduction}

The fungi are saprophytic and parasitic eukaryotic organisms. Historically, the fungi were regarded as relatively insignificant causes of infection. However, the frequency of fungal infections, especially of Invasive Fungal Infections (IFIs), has risen dramatically in recent years due to

Prolonged antibiotic therapy esp Candida spp and Aspergillus

AIDS esp Candida, Cryptococcus, Aspergillus, Histoplasma

Corticosteroid and cytotoxic chemotherapy esp Candida and Aspergillus

I/V catheters and surgery Candida, Aspergillus and Zygomycetes

Opportunistic IFIs remain an important cause of morbidity and mortality. Early and accurate diagnosis is important for timely implementation of antifungal therapy and decreasing the unnecessary use of toxic antifungal agents. In addition the availability of accurate and timely diagnosis could reduce the use of empirical anti-fungal therapy, thereby decreasing antifungal selection pressure and the emergence of antifungal resistance. Standard approaches to the laboratory diagnosis of IFIs include direct microscopic examination in freshly obtained samples, histopathological demonstration of fungi within tissue sections, culture of the causative fungus and its further identification. However, these approaches are often not sufficiently sensitive and/or specific to diagnose IFIs, and they sometimes require invasive procedures to obtain the necessary specimens and culture takes 2-4 wks to become positive. So there is need for rapid methods which are sensitive and specific for diagnosis of fungal infections $[1,2]$. This is a brief review of all the microbiological techniquesconventional and recent alternative techniques especially elaborating antigen detection and molecular techniques which are available for diagnosis of fungal infections.

\section{Diagnostic Methods for Fungal Infections}

Direct examination - wet mount, HPE, flourescent staining

Fungal culture

Radiology

Non culture methods:

a) Serological methods- antigen detection, antibody detection

b) Tests for detection of metabolites

c) Tests for detection of CMI (cell mediated immunity)

d) Molecular methods

e) Others- MALDI-TOF MS

\section{Direct Microscopic Examination of Clinical Specimen}

Microscopy techniques include fresh and stained examination of microbiological samples like $\mathrm{KOH}$, India ink, and calcoflour white stain; and other staining techniques such as Giemsa and periodic acidSchiff, as well as histopathological studies. It is a valuable tool for diagnosis of fungal infections due to following reasons:

A tentative diagnosis can be made before the growth of fungal cells in culture and therefore appropriate antifungal drugs can be initiated.

Observing fungal cells in a clinical specimen may be more valuable as a criterion for diagnosis than isolating in a culture.

These diagnostic methods have important limitations, one being their low sensitivity, so the fungal element is seen only when they are very abundant in the sample to be analysed i.e. when the infection is at an advanced stage. Also it is not possible to identify the species causing 
the infection by means of microscopy examination. Therefore, microscopy cannot be considered to be an early diagnostic technique, however, in many cases, it is the only technique that makes it possible to detect infection $[3,4]$.

$\mathrm{KOH}$ clears the tissue and cellular debris from all types of clinical specimens without damaging the fungal cells. $\mathrm{KOH}$ preparation may help in detecting the presence of yeast forms (budding yeast cells and pseudohyphae) or mycelial fungi in the clinical sample [3]. The presence of pseudohyphae shows colonization and tissue invasion and so their demonstration is significant. Hyphal forms can also be differentiated as hyalohyphomycosis, phaeohyphomycosis or zygomycosis on direct microscopic examination. Zygomycetes have wide, irregular, hyaline hyphae with no septa, and with wide angle branching. Hyalohyphomycetes have fine, hyaline, septate hyphae with acute angle branches, while phaeohyphomycetes have thick-walled filaments with dark pigmentation [4].

Calcoflour White can be added with $\mathrm{KOH}$, which binds specifically to $\beta$ 1-3 D-glucan and gives brilliant apple-green fluorescence when exposed to the shorter wavelengths of ultraviolet light. The disadvantages of using Calcoflour are the need for a Fluorescence microscope, the inability of the dye to detect the endospores within a spherule of Coccidioides immitis, and the difficulty in interpreting vaginal secretions [3].

India ink is a negative staining technique, useful for indicating the presence or absence of extra cellular polysaccharide capsule of fungal cells. The presence of encapsulated yeast cells in CSF in almost always an indicator of Cryptococcal meningitis. Cryptococcal antigen gives false-positive results, hence India ink can be used to complement cryptococcal antigen [3].

Histopathological sections stained with haematoxylin and eosin, periodic acid-Schiff and silver stains, may help in detecting the presence of fungal element in many cases of deep mycosis. Histological sections can also be used to apply immunohistochemical techniques that make it possible to identify species present in tissue using a specific fluorescent antibody that binds to fungal elements in infected tissue. Furthermore, in situ hybridization and nucleic acid amplification methods can also be used, subsequent to the extraction of nucleic acid from tissue [4].

Gormori's Methenamine Silver Stain: It is based on the liberation of aldehyde groups and their subsequent identification by the reduced silver method. It is used for demonstration of polysaccharide content on the fungus in tissue sections. The aldehydes reduce the methenemine silver nitrate complex, resulting in brown black staining fungal cell wall due to the deposition of reduced silver wherever aldehydes are located [3].The fungi and bacteria are stained black, mucopolysaccaride dark grey, cytoplasm old rose and the tissue pale green. It stains the higher bacteria (Nocardia and Actinomycosis) also which are not stained by other fungal stains.

\section{Microbiological Culture}

Culture media which are commonly used for fungal growth include blood agar, Sabouraud dextrose agar with or without cycloheximide, malt extract agar, cornmeal agar, and brain heart infusion agar $[5,6]$. Cultures are incubated at $25^{\circ} \mathrm{C}$ and $37^{\circ} \mathrm{C}$. In samples in which there may be a polymicrobial infection or commensal bacterial flora, selective media are recommended, e.g. Sabouraud dextrose agar containing antibiotics such as chloramphenicol and gentamicin. Some rare mould fungi, such as Fusarium spp. and Scedosporium spp., may be isolated from blood cultures and should not be considered as contaminants. The presence of filamentous fungi merits careful assessment. They are habitual laboratory contaminants and are part of the human saprophytic flora, and they decrease culture specificity. Hence we must consider whether the patient has risk factors for an invasive mycosis and whether fungi have been cultured from other sites in the same patient. Characterization to species level is essential for all clinical isolates collected from deep tissue sites and can be done using conventional methods of classification based on morphological features like chlamydospore formation, germ tube formation, adhesive tape preparation and slide culture and biochemical features like sugar fermentation, sugar assimilation and urease test $[3,6]$.

\section{Radiology}

High-resolution CT and Magnetic Resonance Imaging (MRI) scans make it possible to detect both pulmonary and extrapulmonary invasive aspergillosis and other IFIs. Most patients with IFIs have macronodules and many also have halo signs. Other imaging findings are less common, and these include consolidation, wedge-shaped nodules, cavitary lesions and air-crescent signs [7].

\section{Non-Culture-Based Diagnostic Methods}

Because of the limitations of conventional methods, alternatives to culture techniques have been developed to try to diagnose Invasive Fungal Infections (IFIs) earlier, by detection of fungal cell components. In the 1970s, a method to detect the capsular antigen of Cryptococcus was developed. There have been significant advances in the last few years in alternative methods for diagnosis of fungal infections.

\section{Serological Diagnosis}

Serological diagnosis can be done on the basis of detection of antibodies or antigen in the serum. Antibody detection is useful is assessing prognosis of fungal disease and response to therapy. However, specificity and sensitivity of this is low due to high cross reactions due to complex nature of antigens, also antibodies may be present in the absence of infection due to previous exposure to fungus as a saprophyte or as a commensal. Little or no detectable immune response may be seen in early stage or when cell mediated immunity is depressed or if the antigen is poor immunogen. Hence to overcome these problems, antigen detection is a good alternative. Ideal antigenic markers for invasive fungal infections should not be present too transiently, associated with infection rather than colonization, present sufficiently to diagnose early for the starting of antifungal therapy and should not cross-react with other human and microbial antigens. Various serological techniques used are latex particle agglutination, immunodiffusion, countercurrent immunoelectrophoresis, immunofluorescent antibody, ELISA and Western blotting/ immunoblotting. Important antigens are briefly described below:

\section{Galactomannan Detection}

GM is a component of the cell wall of Aspergillus spp. Many other filamentous fungi, such as Paecilomyces spp. and Penicillium spp., may have GM in their cell wall, but in lower amounts than Aspergillus; hence, quantification of GM is considered specific for diagnosis of aspergillosis [8]. It has high diagnostic value when combined with high-resolution CT, in the early diagnosis of invasive aspergillosis in 
high-risk patients with oncohematological diseases. GM quantification in various samples is considered to be a useful diagnostic tool in paediatric patients, and in follow-up of a patient. A sandwich ELISA that employs rat monoclonal antibody EB-A2 and is known as Platelia Aspergillus (Bio-Rad, France). GM determination in bronchoalveolar lavage (BAL) may be useful in both critically ill patients and solid organ transplant recipients. It has been shown that prior administration of antifungals, especially prophylaxis with itraconazole, decreases test sensitivity. 38 Possible causes of false-positive results are cross-reactions with other fungal species, use of b-lactam antibiotics, children colonized by Bifidobacterium spp. and in neonates, immunoglobulins, haemoderivates, and immunosuppressive drugs such as cyclophosphamide $[9,10]$.

\section{1,3-B-D-glucan detection}

$1,3-\beta$-D-glucan (BG), a cell-wall component of most fungal species, can be detected in blood during IFIs. The BG test (Fungitell, Associates of Cape Cod Inc., USA and Fungitec-G assay), is used as a diagnostic tool for the detection of a broad spectrum of fungal pathogens, with the exception of Mucorales and Cryptococcus neoformans. This test is currently used in combination with classical clinical, radiological and microbiological findings for early diagnosis of aspergillosis in patients with blood diseases; BG quantification has been used for the diagnosis of aspergillosis and other mycoses, such as candidiasis, in critically ill patients and in cases of Pneumocystis pneumonia [11]. However, despite the excellent specificity of the assay, the sensitivity of BG remains low, and a negative result cannot rule out a diagnosis of fungal disease. The technique was of greater use in patients who did not have haematological diseases, although the use of antifungal prophylactic or empirical treatment did not significantly decrease test sensitivity. The use of albumin, immunoglobulins or haemodialysis was associated with false-positive results $[11,12]$.

\section{Mannan Detection}

Mannan is a major component of the Candida cell wall that induces a strong antibody response. Therefore some authors have suggested that the combined detection of mannan (Platelia Candida Ag, Bio-Rad Laboratories) and anti-mannan antibodies (Platelia Candida Ab, BioRad Laboratories) considerably improves the diagnosis of candidemia. While individual sensitivities of these tests is low $(<50 \%)$, the combined detection increases the sensitivity $(60-89 \%)[2,13]$.

\section{Enolase}

Enolase is produced by all Candida spp. except $C$. glabrata. However it has low sensitivity of $54 \%$ and is not available in market.

\section{Secreted Aspartyl Proteinase (Sap)}

Extracellular production of Sap (extracellularly secreted enzyme) by candida is inducible, since Sap has been shown to be produced during active tissue invasion. Hence its extracellular concentration correlates with invasive disease rather than simple colonization. It can be detected by monoclonal antibody based ELISA inhibition technique for C. albicans with sensitivity of $93.9 \%$ and specificity of $96 \%$ [14].

\section{Detection of Capsular Antigen in Cryptococcosis}

It is the most valuable rapid serological test for diagnosis of cryptococcosis. Titre of antigen is highest in serum followed by CSF and urine. Titre in latex agglutination (LA) $\geq 1: 8$ are considered significant. In LA, false positive results are high due to rheumatoid factor, disseminated trichosporonosis, stomatococcus. False negative result seen in prozone like effect. Murex LA (Monoclonal IgM based), eliminates false positive, but has lower sensitivity than LA (Poly IgG). In EIA -sensitivity and specificity similar to LA (Poly IgG), few false positive, not react with rheumatoid factor; also multiple samples can be tested [15].

\section{Antibody Detection in Histoplasmosis}

Histoplasmin contains $H$. capsulatum species-specific $\mathrm{H}$ and $\mathrm{M}$ antigens as well as $\mathrm{C}$ antigen (carbohydrate antigens crossreacts with coccidioides species and B.dermatitidis). Antibodies against $\mathrm{H}$ antigen ( $\mathrm{H}$ band fine line, Present near well containing antibodies) form during active histoplasmosis, while antibodies to $M(M$ band) antigens may be formed in active and chronic histoplasmosis and are usually the first to arise upon seroconversion. This is done using immunodiffusion test, with commonly used titre of 1:32, or 4 fold rise in antibody titre is considered significant $[3,4]$.

\section{Test for Detection of Metabolites}

This is to demonstrate a distinctive fungal metabolic byproduct in the body fluids of patients. Metabolites like D-Arabinitol are produced in large amount by all strains of $C$. albicans, $C$. tropicalis, $C$. parapsilosis, whereas no strain of $C$. glabrata, C. krusei, or $C$. neoformans produce $\mathrm{D}$-arabinitol. It can be used as serum marker for diagnosis of candida spp. and also as prognostic indicator. Production of D-Arabinitol is detected by Gas chromatography (GC) in serum and/or urine or by quantifying D-arabinitol enzymatically and detected spectrophotometrically [3].

\section{Tests for detecting CMI}

Skin testing can be used for establishing etiological diagnosis especially in nonendemic area, for epidemiological surveys and as prognostic indicator, as a positive skin test may revert back to negative with severe or disseminated diseases. Skin testing is done in Aspergillus fumigates, Histoplasma capsulatum, Sporothrix schenkii and others. However it can give false positive reaction in endemic regions and because of cross reactions due to sharing of antigens. False negative can occur due to early stage of infection and defective CMI $[3,4]$.

\section{Nucleic acid Detection}

Fungal nucleic acid detection in clinical samples has great potential to amplify small quantities of DNA. Molecular identification, based on the sequencing of several DNA targets or fragments, has shown that classification by conventional techniques is not reliable for some moulds, especially the less common species [16]. Molecular identification should be considered, usually by referral to a reference laboratory, for cases that require confirmation or a more exhaustive analysis of the causative agent. Detection and identification of fungal pathogens by DNA-based methods can yield results sooner than cultivation and also the sensitivity and specificity are higher than cultures and serologic tests for diagnosing IFIs. Also DNA results have correlated with clinical improvement and effect of treatment, in patients with invasive aspergillosis, invasive candidiasis, and cryptococcal meningitis. Furthermore, this strategy made it possible to decrease the administration of antifungal drugs by reducing their 
empirical use [17]. However there are certain disadvantages associated with molecular techniques:

Molecular diagnostic methods may not distinguish colonization from infection

False-positive results of $31 \%$ and ranging from 8 to $38 \%$ have been reported in the diagnosis of candidiasis and aspergillosis due to colonization of the patients' airway by conidia and/or hyphae and the contamination of sample and/or reaction buffers by environmental fungi.

False-negative results of $21 \%$ of patients with positive blood cultures for Candida species, due to the low sensitivity of detection of single-copy gene, hence it is necessary for these techniques to incorporate an internal control.

There are some commercial molecular methods for fungal detection: (i) SeptiFast diagnostic kit (Roche Molecular Diagnostics, USA), which detects up to 25 micro-organisms including five Candida species and Aspergillus fumigatus, and (ii) MycAssay kits (Myconostica Ltd, UK), one of which detects Aspergillus DNA (15 species) in serum or BAL, and one of which detects $P$. jiroveci DNA in respiratory samples. Sensitivities range from $78 \%$ to $100 \%$ for candidiasis and from $33 \%$ to $100 \%$ in patients with proven invasive aspergillosis. Work has been performed with PCR techniques with other mould species such as Zygomycetes, Scedosporium spp., Fusarium spp. and endemic fungi $[1,2]$. In the last decade, effective amplification platforms, probe development and various quantitative PCR technologies have revolutionised research on fungal detection. Some of the latest techniques employed in the detection of fungi, including fluorescence in situ hybridisation (FISH), DNA array technology, Multiplex tandem PCR, Real time PCR, PCR- ELISA, RAPD and Loop-Mediated Isothermal Amplification (LAMP).

\section{Fluorescent in situ Hybridization (FISH)}

FISH is a powerful method for the in situ detection of active growing organisms in environmental samples. It can visualize the precise location of particular DNA or RNA sequences in the cytoplasm, organelles, or nuclei of biological materials. FISH probes often target sequences of ribosomal RNA or mitochondrial genes because they are abundant in sequence databases and in multiple copies in each cell. Limitations of the FISH method can include fungal and substrate inherent autofluorescence, insufficient permeability of cell walls, non-specific binding of probes, and low ribosome contents. PNA (peptide nucleic acid) probes are mimics in which the negatively charged sugar-backbone of DNA is replaced with a non-charged polyamide backbone. PNA probes penetrate cell walls more effectively due to their neutrality and they do not have to overcome the destabilizing electrostatic repulsion during hybridisation [18,19].

\section{DNA array Hybridization}

DNA array hybridization, also known as Reverse Dot Blot Hybridization (RDBH) or macroarray, is a technique based on hybridization of amplified and labelled genome regions of interest to immobilized oligonucleotides spotted on a solid support platform. It is considered a powerful and practical technique for the detection and identification of fungi and other microbes, such as bacteria, from complex environmental samples without the need for isolation in culture [20,21]. Synthesized oligonucleotides are spotted onto a supporting platform, such as a nylon membrane or glass slide, either manually or robotically. A positive reaction between an amplicon and a perfectly matched (PM) oligonucleotide generates a chemiluminescent signal which can be detected by a digital camera in dark rooms. With the unlimited capacity for the accommodation of oligonucleotides on one membrane and the reusability of the membranes, it shows superior multiplexing detection capability at a lower cost over other PCR-based methods [20].

\section{Multiplex tandem PCR (MT-PCR)}

MT-PCR is a technology platform developed for highly multiplexed gene expression profiling and the rapid identification of clinically important pathogens. The platform consists of two rounds of amplification. In the first step, a multiplex PCR is performed at 10 to 15 cycles to allow enrichment of target DNA without creating competition between amplicons [21]. This product is used as template for the second amplification that consists of multiple individual quantitative PCR reactions with primers nested within those used in the multiplex PCR. Up to 72 different PCR reactions can be multiplexed and performed simultaneously. The incorporation of two sets of species-specific primers for each target ensures correct amplification and detection, thus avoiding the expense of DNA probes. MT-PCR is considered a suitable alternative for the rapid detection and identification of fungal pathogens directly from clinical specimens, thus circumventing the need for gold standard culture and histology, which is slow, insensitive and non-specific. AusDiagnostics (Alexandria, NSW, Australia), developed several MT-PCR assays to detect the16 major causes of fungal bloodstream infections.

\section{Real time PCR}

It combines PCR, probe hybridization, and signal generation in one step. The TaqMan probe consists of a reporter dye with a fluorescein derivative at the $5^{\prime}$ end, a 3 ' quencher dye, and a 3 ' blocking phosphate group. Increase in reporter dye fluorescence, resulting from the cleavage between the reporter and quencher, occurs. The amount of reporter dye released is proportional to the amount of DNA amplified by PCR. The TaqMan fluorescence assay enables samples to be analyzed as soon as 5 to $10 \mathrm{~min}$ after PCR is complete, and no postamplification manipulation is required, which might reduce a significant source of laboratory contamination. It is 10 -fold more sensitive than detection by ethidium bromide-stained agarose gel. TaqMan assays are available to detect isolates of Candida species and Aspergillus fumigates [15].

\section{PCR-ELISA}

A three-part method which included PCR amplification, hybridization with the complementary labeled probe and detection of reaction products in an EIA that provides either colorimetric or fluorescence readout. The sensitivity of PCR-EIA to detect candidemia and aspergillosis was higher than that by ethidium bromide staining and multiple samples can be assayed in parallel.

\section{Random Amplified Polymorphic DNA (RAPD)}

It is a type of PCR reaction, but the segments of DNA that are amplified are random. By resolving the resulting patterns, a semiunique profile can be generated from a RAPD reaction. Differentiation between Cryptococcus gatti and Cryptococcus neoformans is possible by RAPD. 


\section{Loop Mediated Isothermal Amplification (LAMP)}

LAMP is a powerful and novel nucleic acid amplification method that amplifies a few copies of target DNA with high specificity, efficiency, and rapidity under isothermal conditions (do not require a thermal cycler), using a set of four specially designed primers and a DNA polymerase with strand displacement activity [22,23]. The cycling reactions can result in the accumulation of $10^{9}$ to $10^{10}$-fold copies of target in less than an hour. Considering the advantages of rapid amplification, simple operation and easy detection, LAMP has potential applications for clinical diagnosis as well as surveillance of infectious diseases in developing countries without requiring sophisticated equipment or skilled personnel [22]. LAMP products can be directly observed by the naked eye (SYBR Green I stain) or under UV illumination, the gel shows a ladder-like structure from the minimum length of target DNA up to the loading well, which are the various length stem-loop products of the LAMP reaction. LAMP assays have been developed for the rapid detection of pathogenic fungi like Penicillium marneffei, Fonsecaea agents of chromoblastomycosis, Cryptococcus spp., Pseudallescheria and Scedosporium species.

\section{Other Techniques}

\section{MALDI-TOF MS}

Matrix-assisted laser desorption/ionization -time of flight mass spectrometry (MALDI-TOF MS), a Noble prize winning technology is making a revolutionary entry in clinical microbiology laboratory laboratories all over the world. Although most studies have been carried out with bacteria, publications on the utility of this method for rapid and reliable identification of fungi of medical importance are increasing. The methodology of identification of yeasts and filamentous fungi by MALDI-TOF MS is still evolving. Most species of Candida and filamentous fungi such as Aspergillus species can be identified rapidly and accurately by this technique [24].

\section{Conclusion}

Invasive fungal infections constitute a major challenge for the management of immunocompromised patients, mainly haematooncology patients, transplant etc. Conventional microbiological, histological and radiological techniques remain the cornerstone of diagnosis but are insensitive and have a limited impact on clinical decision-making. There is always a need to characterize fungi quickly and accurately which can be done by rapid diagnostic techniques like antigen detection and molecular techniques. Molecular techniques favor early identification of microorganisms, thereby enhancing epidemiological studies as well as the diagnosis of fungi, which is essential to the recognition of pathogens and its appropriate treatment. Despite many novel technologies being available, challenges remain to identify as yet unculturable fungi, to determine cryptic species and it is also necessary to determine sensitivity, specificity, accuracy, frequency of testing and cost of these tests.

\section{References}

1. Cuenca-Estrella M, Bassetti M, Lass-Flörl C, Rácil Z, Richardson M, et al. (2011) Detection and investigation of invasive mould disease. J Antimicrob Chemother 66 Suppl 1: i15-24.

2. Yeo SF, Wong B (2002) Current status of nonculture methods for diagnosis of invasive fungal infections. Clin Microbiol Rev 15: 465-484.
3. Bennett, Chang (1995) Laboratory Aspects of Medical Mycology, Laboratory diagnosis: 45-78.

4. Diagnostic manual, procedures in mycology, Chapter XIV page 109-132.

5. Gadea I, Cuenca-Estrella M, Martín E, Pemán J, Pontón J, et al. (2007) [Microbiological procedures for diagnosing mycoses and for antifungal susceptibility testing]. Enferm Infecc Microbiol Clin 25: 336-340.

6. Hoog GS, Guarro J, Gene J (2000) Atlas of Clinical Fungi. Utrecht/ Reus: Centraal bureau voor Schimmelcultures/Universitat Rovira i Virgili.

7. de Pauw B, Walsh TJ, Donnelly JP (2008) Revised definitions of invasive fungal disease from the European Organization for Research and Treatment of Cancer/Invasive Fungal Infections Cooperative Group and the National Institute of Allergy and Infectious Diseases Mycoses Study Group (EORTC/MSG) Consensus Group. Clin Infect Dis 46: 1813-1821.

8. Aquino VR, Goldani LZ, Pasqualotto AC (2007) Update on the contribution of galactomannan for the diagnosis of invasive aspergillosis. Mycopathologia 163: 191-202.

9. Racil Z, Kocmanova I, Lengerova M, Winterova J, Mayer J (2007) Intravenous PLASMA-LYTE as a major cause of false-positive results of platelia Aspergillus test for galactomannan detection in serum. J Clin Microbiol 45: 3141-3142.

10. Koo S, Bryar JM, Page JH, Baden LR, Marty FM (2009) Diagnostic performance of the (1-->3)-beta-D-glucan assay for invasive fungal disease. Clin Infect Dis 49: 1650-1659.

11. Obayashi T, Negishi K, Suzuki T, Funata N (2008) Reappraisal of the serum (1-->3)-beta-D-glucan assay for the diagnosis of invasive fungal infections--a study based on autopsy cases from 6 years. Clin Infect Dis 46: $1864-1870$.

12. Cuenca-Estrella M, Bassetti M, Lass-Flörl C, Rácil Z, Richardson M, et al. (2011) Detection and investigation of invasive mould disease. J Antimicrob Chemother 66 Suppl 1: i15-24.

13. Naglik JR, Challacombe SJ, Hube B (2003) Candida albicans secreted aspartyl proteinases in virulence and pathogenesis. Microbiol Mol Biol Rev 67: 400-428, table of contents.

14. Collazos J (2003) Opportunistic infections of the CNS in patients with AIDS: diagnosis and management. CNS Drugs 17: 869-887.

15. Valones MA, Guimarães RL, Brandão LA, de Souza PR, de Albuquerque Tavares Carvalho A, et al. (2009) Principles and applications of polymerase chain reaction in medical diagnostic fields: a review. Braz J Microbiol 40: 1-11.

16. Barnes RA, White PL, Bygrave C, Evans N, Healy B, et al. (2009) Clinical impact of enhanced diagnosis of invasive fungal disease in high-risk haematology and stem cell transplant patients. J Clin Pathol 62: 64-69.

17. Wilson DA, Joyce MJ, Hall LS, Reller LB, Roberts GD, Hall GS, et al (2005) Multicenter evaluation of a Candida albicans peptide nucleic acid fluorescent in situ hybridization probe for characterization of yeast isolates from blood cultures. Journal of Clinical Microbiology 43: 29092912.

18. Shepard JR, Addison RM, Alexander BD, Della-Latta P, Gherna M, Haase $G$ et al (2008) Multicenter evaluation of the Candida albicans/Candida glabrata peptide nucleic acid fluorescent in situ hybridization method for simultaneous dual-color identification of C. albicans and C. glabrata directly from blood culture bottles. Journal of Clinical Microbiology 46: 50-55.

19. Chen W, Seifert K, Lévesque CA (2009) A high density COX1 barcode oligonucleotide array for identification and detection of species of Penicillium subgenus Penicillium. Molecular Ecology Resources 9: 114129.

20. Zhang N, McCarthy ML, Smart CD (2008) A macroarray system for the detection of fungal and oomycete pathogens of solanaceous crops. Plant disease 92: 953-960.

21. Lau A, Chen S, Sleiman S, Sorrell T (2009) Current status and future perspectives on molecular and serological methods in diagnostic mycology. Future Microbiol 4: 1185-1222.

22. Parida M, Sannarangaiah S, Dash PK, Rao PV, Morita K (2008) Loop mediated isothermal amplification (LAMP): a new generation of 
Citation: Malhotra S, Sharma S, Bhatia NJK, Kumar P, Bhatia NK, et al. (2014) Recent Diagnostic Techniques in Mycology. J Med Microb Diagn 3: 146. doi:10.4172/2161-0703.1000146

Page 6 of 6

innovative gene amplification technique; perspectives in clinical diagnosis of infectious diseases. Reviews in Medical Virology 18: 407421.

23. Tomita N, Mori Y, Kanda H, Notomi T (2008) Loop-mediated isothermal amplification (LAMP) of gene sequences and simple visual detection of products. Nat Protoc 3: 877-882.
24. Brun S, Madrid H, Gerrits Van Den Ende B, Andersen B, MarinachPatrice C, et al. (2013) Multilocus phylogeny and MALDI-TOF analysis of the plant pathogenic species Alternaria dauci and relatives. Fungal Biol 117: 32-40. 